\title{
LA NUEVA DISPLASIA BRONCOPULMONAR DESDE EL PUNTO DE VISTA DEL NEUMÓLOGO PEDIATRA
}

\author{
New bronchopulmonary dysplasia, from a pediatric pulmonologist's perspective
}

Dra. Marcela B Linares $P$

Pediatra Especialista en Enfermedades Respiratorias

Docente Universidad Finis Terrae

Clínica Indisa

NEW BRONCHOPULMONARY DYSPLASIA, FROM A PEDIATRIC PULMONOLOGIST'S PERSPECTIVE

Bronchopulmonary dysplasia (BPD) is the most prevalent chronic lung disease of prematurity. The so-called new BPD has replaced the classic BPD described by Northway, as a result of maternal use of corticosteroids, early surfactant and less aggressive mechanical ventilation and the survival of younger premature, born during the canalicular stage and that completed their alveolization outside the uterus. The new BPD is a less severe disease, but lung function is impaired in the long-term. An update of the new BPD, focused on the management after discharge from neonatology, from a pediatric pulmonologist perspective is presented.

Key words: alveolarization, prematurity, chronic lung disease, therapy, prognosis

\section{RESUMEN}

La Displasia Broncopulmonar (DBP) es la enfermedad pulmonar crónica más prevalente del prematuro. La denominada nueva DBP ha reemplazado a la DBP clásica descripta por Northway, como consecuencia del uso de corticoides maternos, surfactante precoz, ventilación mecánica menos agresiva y la sobrevivencia de prematuros más pequeños, que nacen en etapa canalicular de su desarrollo pulmonar y completan su alveolización fuera del útero. La nueva DBP es una patología menos severa, pero con compromiso funcional respiratorio a largo plazo. A continuación se describe una actualización de la nueva DBP, enfocada en el manejo realizado luego del alta de neonatología, desde el punto de vista del Neumólogo Pediatra.

Palabras clave: alveolarización, prematuro, displasia broncopulmonar, terapia, pronóstico

\section{INTRODUCCIÓN}

La displasia broncopulmonar (DBP) es una enfermedad pulmonar crónica que se produce como consecuencia de múltiples factores que intervienen en la vía aérea inmadura del recién nacido prematuro, provocando una disminución del crecimiento pulmonar, dando lugar a una limitación en la función respiratoria de grado variable (1).

Es la enfermedad pulmonar crónica que con más frecuencia afecta al recién nacido prematuro. La incidencia de la DBP es variable entre los distintos centros neonatológicos del país, fluctuando entre un $20 \%$ y un $35 \%$ en los menores de 1500 g y alcanza un $60 \%$ en los menores de $1000 \mathrm{~g}$ (2).

A pesar de los avances en la atención perinatal de los prematuros y la declinación de la tasa de mortalidad entre los recién nacidos de muy bajo peso al nacer (menor a 1500 gramos), no ha habido una disminución de la incidencia de la

\section{Correspondencia:}

Dra Marcela Linares

Clínica Indisa,

Los Españoles 1855, Providencia

e-mail:m.linares@manquehue.net
DBP, aunque si se ha manifestado un cambio en la expresión clínica y la gravedad de esta patología, lo que constituye la nueva DBP $(1,3,4)$.

\section{ETIOLOGÍA Y FISIOPATOGENIA}

La DBP fue descripta por Northway en 1967, en prematuros de 34 semanas con distress respiratorio, sometidos a altas concentraciones de oxígeno y altos parámetros ventilatorios (5). Desde aquella época ha habido un incremento en la sobrevida de prematuros más pequeños, además de una reducción en las secuelas. Este cambio se debió al uso de esteroides maternos prenatales y de surfactante en el prematuro después de su introducción en los años 80 , lo que ha permitido disminuir el distress respiratorio y la mortalidad, pero no la incidencia de DBP. Esto, sumado al empleo de menores concentraciones de oxígeno y ventilación menos agresiva, ha traído como consecuencia la aparición de la nueva DBP, de la era postsurfactante, en la que sobreviven prematuros que se encuentran en el período canalicular de su desarrollo pulmonar (antes de la semana 26 de gestación), el cual es completado fuera del útero, produciéndose un freno en la alveolarización y 
la angiogénesis del pulmón y llevando a una menor superficie de intercambio (6)

A pesar que la edad gestacional y el crecimiento intrauterino son predictores del desarrollo de DBP, no hay un factor etiológico único que explique su incidencia y severidad (4). Se han descripto factores prenatales, postnatales, genéticos y epigenéticos que influyen en la expresión de la DBP. Dentro de los factores prenatales se describe el tabaquismo materno, la preeclampsia, corioamionitis y colonización con Ureaplasma. Entre los mecanismos postnatales se han descripto la persistencia del ductus, el barotrauma, volutrauma e hiperoxia. Estos factores actúan sobre el prematuro, quien en algunos casos puede tener predisposición genética, bajas concentraciones de antioxidantes y un pulmón que aún no ha comenzado su alveolarización. Como consecuencia de la interacción de estos factores, se produce una disminución del factor de crecimiento endotelial vascular (VEGF, vascular endothelial growth factor), aumento de citoquinas y factores inflamatorios, disminución del factor de crecimiento fibroblástico y formación de radicales libres con efecto oxidativo, lo que determina un lecho vascular pulmonar hipoplásico e irregularmente distribuido y reducción en la alveolización, ya que la vía aérea y el lecho vascular del pulmón del feto, retroalimentan su desarrollo con la intervención del VEGF (Figura1) $(6,7)$.

\section{Factores ambientales}

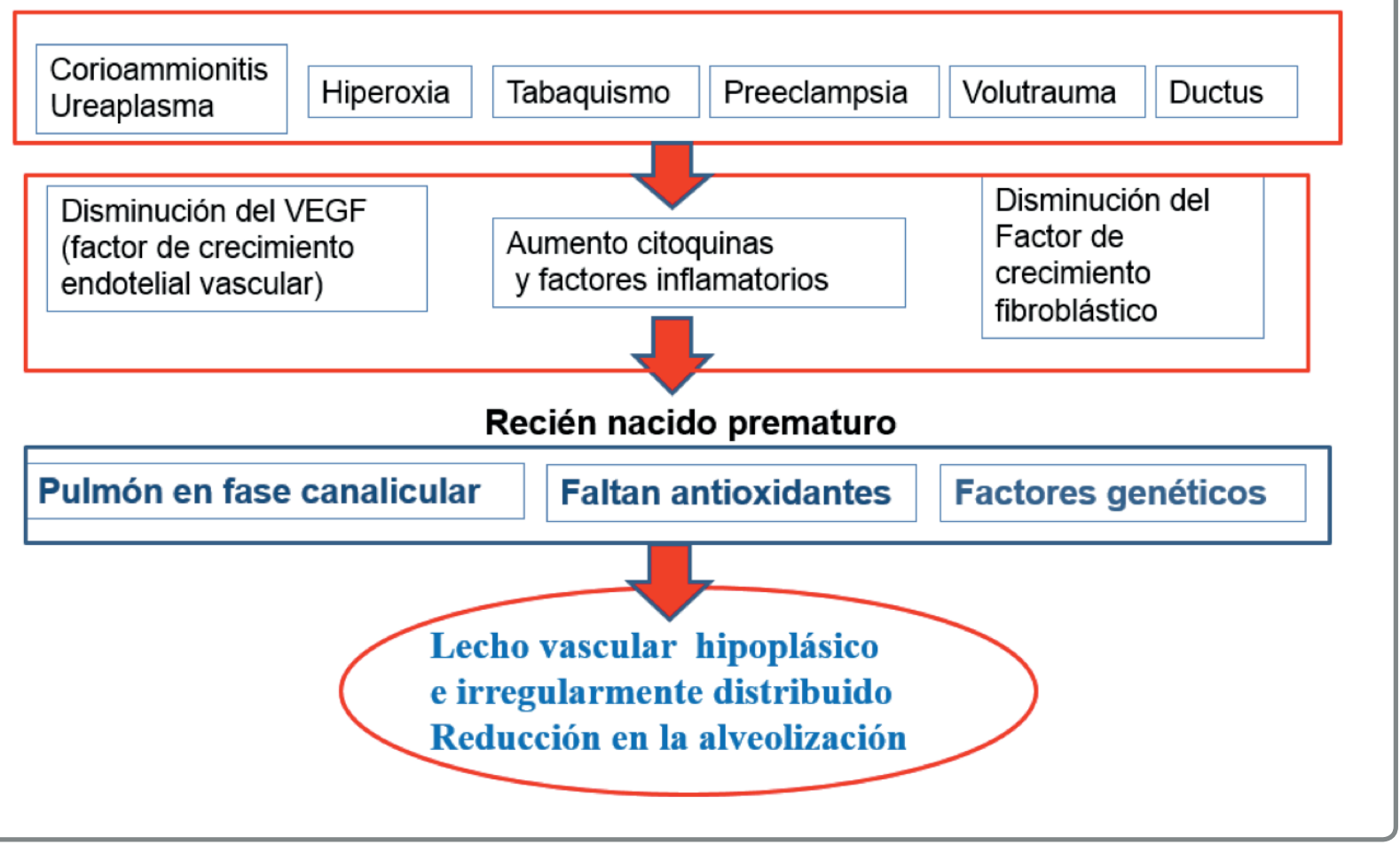

Figura 1. Factores que intervienen en el desarrollo de Displasia Broncopulmonar.

En la actualidad no se conoce asociación genómica con el desarrollo de DBP, solo hay estudios que sugieren la participación de potenciales genes modificadores relacionados con la codificación de la proteína del surfactante, las citoquinas y los factores de crecimiento endotelial (6). Recientemente se ha estudiado el rol del mecanismo epigenético, el que se define como la influencia de factores ambientales en la expresión genética, ocasionando una reprogramación en la secuencia de bases. Por ejemplo, un aumento en la tensión de 02 en el alvéolo causaría mecanismos epigenéticos que alteran la transcripción de la proteína A del surfactante (6).

\section{ANATOMÍA PATOLÓGICA Y CONSECUENCIAS FISIOPATOLÓGICAS}

Las características anatomopatológicas de la nueva DBP, descritas en la Tabla 1, en comparación con la DBP clásica, se traducen en una disminución de la superficie de intercambio, lo que lleva a una reducción de la tolerancia al esfuerzo y a infecciones respiratorias, predisposición a la hipertensión pulmonar (HTP) y necesidad de oxigenoterapia prolongada $(1,8)$. 


\begin{tabular}{|l|l|}
\hline DBP clásica: lesiones en parche & Nueva DBP: compromiso difuso \\
\hline Fibrosis septal & Menos uniones alveolares \\
\hline Metaplasia escamosa & Menos alvéolos, más simples y distendidos \\
\hline Inflamación y fibrosis de la mucosa & Calibre de vía aérea reducido \\
\hline Atelectasias & Leve inflamación y fibrosis \\
\hline Pérdida de uniones alveolares, enfisema & Lesiones epiteliales leves \\
\hline Cambio vascular hipertensivo & Vasculatura dismórfica \\
\hline
\end{tabular}

Tabla 1. Diferencias anatomopatológicas entre Displasia Broncopulmonar clásica y nueva Displasia

El prematuro puede presentar un pulmón restrictivo, con predominio de hipoplasia pulmonar, u obstructivo con diferentes grados de hiperreactividad bronquial (HRB) debido a una vía aérea más pequeña. Generalmente se encuentran ambos componentes.

La incidencia promedio de hipertensión pulmonar en pacientes con DBP es de un 18 a $25 \%$, llegando a un $50 \%$ en los casos más severos. La HTP contribuye significativamente a la morbimortalidad en la DBP (8).

La vía aérea superior puede presentar traqueomalacia, laringomalacia, estenosis subglótica, parálisis unilateral de cuerdas vocales o granulomas en la vía aérea, aunque con menor frecuencia que en la DBP clásica $(9,10)$.

Hay varios órganos involucrados en la fisiopatología de la DBP, los que se afectan como consecuencia de la DBP y de la prematurez, y a su vez aumenta el compromiso respiratorio, en un mecanismo de retroalimentación positivo (10) (Figura 2).

El corazón puede presentar sobrecarga derecha por la HTP y disminución del llene del ventrículo izquierdo por desviación del tabique interventricular a la izquierda. El aumento de la presión transpulmonar a su vez puede exacerbar el reflujo gastroesofágico. La disminución de la excreción renal de agua debido a una disminución del gasto cardíaco, lleva a un aumento del edema pulmonar con disminución de la compliance, aumento de la resistencia de vía aérea y aumento de la precarga del ventrículo izquierdo. La hipoxemia puede afectar el crecimiento y desarrollo neurológico y disminución del progreso de peso. La bomba torácica es menos eficiente y aumenta el trabajo ventilatorio, hay mayor utilización de calorías destinadas a la ventilación y no al crecimiento somático. La taquipnea producida por la DBP y el retraso neurológico secundario a la prematurez, pueden desencadenar un trastorno de succión deglución, con mayor probabilidad de aspiración, broncoespasmo y desnutrición. El centro respiratorio puede presentar una reprogramación secundaria a la fatiga muscular respiratoria crónica, predisponiendo a trastornos respiratorios durante el sueño, incluso apneas obstructivas por hipotonía de la vía aérea superior (10). Todos estos factores, se deben tener en cuenta a la hora de tratar a los prematuros con DBP.

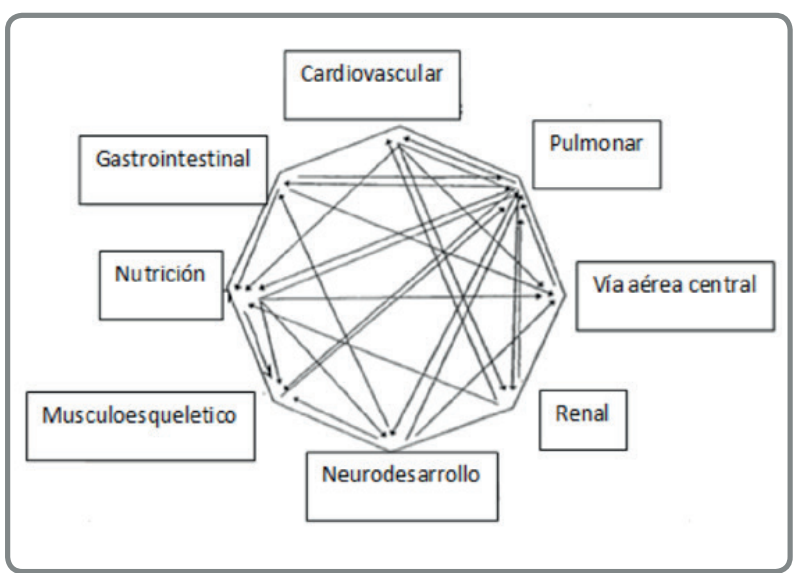

Figura 2. Interrelación del pulmón del prematuro con otros órganos y sistemas

\section{DEFINICIÓN, CLÍNICA Y GRADOS DE SEVERIDAD}

La DBP se define como la necesidad de oxígeno adicional de un prematuro durante al menos 28 días, y el requerimiento de oxígeno a las 36 semanas de edad corregida 0 al alta (en menores de 32 semanas al nacer), 0 entre los 28 y 56 días de vida 0 al alta (en mayores de 32 semanas al nacer) (Tabla 2) (11). Estas definiciones tienen diferente sensibilidad y especificidad para el diagnóstico de DBP, lo que lleva a incidencias de la enfermedad que varían según la definición utilizada (12). 


\begin{tabular}{|c|c|c|}
\hline Edad gestacional & $<32$ semanas & $\geq 32$ semanas \\
\hline Edad al diagnóstico & $\begin{array}{c}36 \text { semanas edad corregida } 0 \\
\text { alta domiciliaria, lo que se cumpla } \\
\text { primero }\end{array}$ & $\begin{array}{c}>28 \text { días, pero }<56 \text { días de vida } \\
0 \text { alta domiciliaria, lo que se } \\
\text { cumpla primero }\end{array}$ \\
\hline \multicolumn{3}{|c|}{ Tratamiento con $02>21 \%$ por más de 28 días } \\
\hline DBP leve & $\begin{array}{l}\text { Respirando aire ambiental a las } 36 \\
\text { semanas edad corregida o al alta, lo } \\
\text { que se cumpla primero }\end{array}$ & $\begin{array}{l}\text { Respirando aire ambiental a los } \\
56 \text { días de vida o al alta, lo que se } \\
\text { cumpla primero }\end{array}$ \\
\hline DBP moderada & $\begin{array}{l}\text { Necesidad de oxígeno }<30 \% \text { a las } \\
36 \text { semanas de edad corregida o al } \\
\text { alta, lo que se cumpla primero }\end{array}$ & $\begin{array}{c}\text { Necesidad de oxígeno }<30 \% \text { a los } \\
56 \text { días de vida o al alta, lo que se } \\
\text { cumpla primero }\end{array}$ \\
\hline DBP severa & $\begin{array}{l}\text { Necesidad de oxigeno } \geq 30 \% \text { y/o } \\
\text { presión positiva (CPAP o VM) a las } \\
36 \text { semanas edad corregida o al } \\
\text { alta, lo que se cumpla primero }\end{array}$ & $\begin{array}{c}\text { Necesidad de oxígeno } \geq 30 \% \text { y/o } \\
\text { presión positiva (CPAP o VM) a los } \\
56 \text { días de vida o al alta, lo que se } \\
\text { cumpla primero }\end{array}$ \\
\hline
\end{tabular}

Tabla 1. Definición y criterios diagnósticos según NICHD/NHLBI/ORD WORKSHOP $(9,11)$

Si el recién nacido vive sobre el nivel del mar, se debe considerar un factor de corrección que determine la fracción inspirada de oxígeno (FIO2) por encima de la cual se va a definir la DBP. A nivel del mar esta FI02 es de $21 \%$, pero por ejemplo, a una altura de 2400 metros, se requiere una Fi02 mayor de $28 \%$ para ser diagnosticado como DBP (13). Esta hipoxemia relativa se compensa con otros mecanismos que contribuyen a la entrega de oxígeno a los tejidos: aumento de la concentración de hemoglobina, de la frecuencia cardíaca y el volumen de eyección.

Hasta 2/3 de los recién nacidos con la nueva DBP no presentan una enfermedad severa. Varios de ellos pueden no requerir oxigeno las primeras semanas de vida. Esto se debe a la disminución de la superficie de intercambio gaseoso, lo que lleva a una reducción de la reserva pulmonar, poniéndose de manifiesto cuando el prematuro aumenta sus demandas ventilatorias (1).

Los gases en sangre se caracterizan por una acidosis respiratoria compensada. La radiografía de tórax presenta distintos grados de infiltrado reticulonodular difuso bilateral, hiperinsuflación y en algunos casos atelectasias. La radiografía de tórax denota un compromiso difuso y menor que el que se observaba en la antigua DBP (Figura 3)

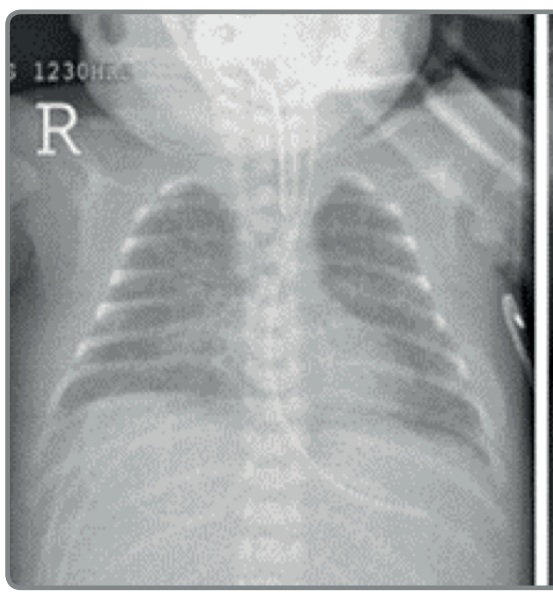

Nueva Displasia

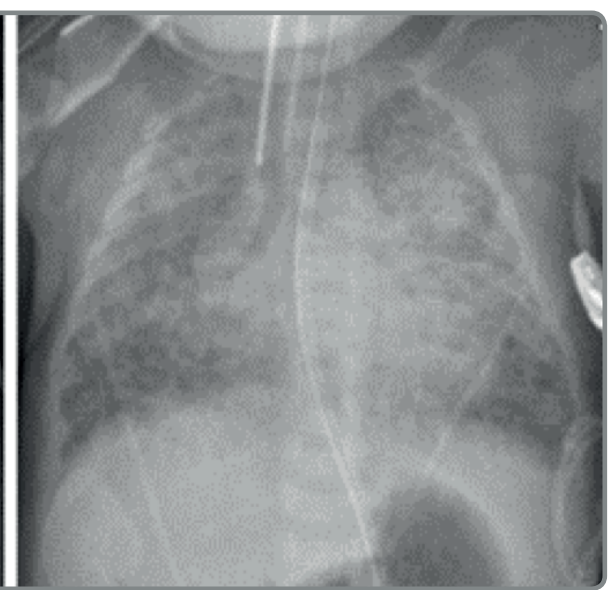

Displasia clásica
Figura 3.

Radiografía de tórax de recién nacido con Displasia Broncopulmonar 


\section{TRATAMIENTO}

Mantener oxigenación y aporte calórico adecuado, que permitan al niño crecer adecuadamente y evitar las sobreinfecciones, son las únicas terapias que han demostrado efectividad. Hay que tratar el reflujo gastroesofágico si se sospecha clínicamente y descartar aspiración (14).

Hay gran discrepancia en los distintos centros, en la definición de oxigenación adecuada en el prematuro, lo que tiene gran relevancia si se tiene en cuenta que determina la definición de la DBP. La falta de consenso surge de confundir al recién nacido prematuro que tiene menos de 34 semanas de vida, que requiere menores concentraciones de oxígeno para evitar complicaciones (15), con el recién nacido prematuro que ya tiene diagnóstico de DBP, que ha estado estable al menos 2 semanas, que tiene más de 34 semanas y esta pronto a su alta, y no tiene riesgos de complicaciones por la oxigenoterapia. Este lactante debe saturar un promedio de al menos 95\% y se deben evitar saturaciones menores a 92\%, lo que va a permitir disminuir el riesgo de muerte, aumentar la ganancia de peso, evitar el desarrollo de hipertensión pulmonar, disminuir la resistencia de la vía aérea y el trabajo respiratorio, mejorar la arquitectura del sueño, la readmisión hospitalaria y el compromiso neurológico (16-18). En el único estudio de saturometría realizado en niños prematuros sin DBP, con tecnología de extracción de señal, se ha observado un promedio de saturación entre 95 y 100\%, con solo un $6 \%(+-6,1)$ del tiempo de 12 horas de registro saturaciones menores a $93 \%$ (19). En recién nacidos a término menores de 5 días de vida, se describe un promedio de saturación de 97,3\% y un índice de desaturación 80\% menor a 1, con una duración menor a 19 segundos en cada episodio (20). Por lo que se sugiere al alta, el oxígeno necesario para saturar un promedio de $95 \%$, y no más de 1 evento por hora de desaturaciones menores a $80 \%$ y menores de 20 segundos de duración.

En los casos más severos, en los que el recién nacido no puede ser destetado del ventilador por la severidad de la displasia o agravado por un daño neurológico, se debe tomar la decisión de realizar traqueostomía. El momento más óptimo de su indicación es entre los 2 y 3 meses de vida, con parámetros ventilatorios estables. Se ha demostrado que al traqueostomizar a esta edad, en la que el lactante ya tiene al menos 40 semanas, permite realizar intervenciones que mejoren su neurodesarrollo, lo que no se puede lograr con el niño con tubo endotraqueal. Además permite un descenso de los parámetros de la ventilación (21).

Algunas terapias que han demostrado algún impacto en prevenir la DBP son la Vitamina A, las xantinas y la azitromicina, aunque no son utilizadas en todos los centros por su alto costo en caso de la Vitamina A y la falta de conocimiento de la farmacocinética y efectos secundarios en el caso de al azitromicina en el neonato $(3,7,22,23)$.

Con respecto a los corticoides sistémicos, Grier y Halliday (24) han demostrado que por cada 100 niños que reciben corticoides en forma precoz, la DBP se puede prevenir en 10 casos, a expensas de 6 niños con hemorragia gastrointestinal,
12 con parálisis cerebral y 14 con examen neurológico anormal durante el seguimiento; por lo que se desaconseja su utilización. En ocasiones pueden utilizarse, previo consentimiento informado, en mayores de 3 semanas, por períodos de no más de 3 a 7 días, para facilitar el retiro del ventilador (25). Los corticoides inhalados no han demostrado beneficios (26). Con respecto a los diuréticos, si bien son muy utilizados, hay poca evidencia de beneficios clínicos y mucha de efectos colaterales (27).

Debido a la falta de reserva pulmonar de los lactantes con DBP, la posibilidad de requerir nuevas hospitalizaciones por infecciones respiratorias es de alrededor de un 60\% durante el 1 er año de vida, bajando a un $20 \%$ en el segundo año (28). Por lo que se debe indicar vacunas con acción capullo: influenza en los convivientes mayores de 6 meses y pertussis acelular a los mayores de 12 años; aislamiento y lavado de manos, no asistir a sala cuna los primeros 2 años de vida, prohibir tabaquismo y calefacción contaminante y promover una atención preferencial en los controles de salud (14).

Para prevenir las infecciones graves por virus respiratorio sincicial (VRS), se debe indicar palivizumab (anticuerpos monoclonales anti VRS) en Chile durante los meses de abril a agosto con un máximo de 5 dosis. Se indica en todos Ios niños con DBP, nacidos antes de las 32 semanas, menores de 1 año y a los que teniendo entre 1 y 2 años de edad están con terapia (oxigeno, diuréticos o corticoides sistémicos) debido a la DBP, dentro de los 6 meses previos al comienzo del período invernal (29). Si el recién nacido con DBP está hospitalizado aún en neonatología en período invernal, se debe colocar la primera dosis 2 o 3 días antes del alta. Si se hospitaliza debido a un cuadro por VRS, no se debe completar la profilaxis (29).

\section{EVOLUCIÓN}

En la mayoría de los casos de DBP moderada, si se evitan las infecciones respiratorias durante los 2 primeros años y el lactante presenta un crecimiento adecuado, la evolución es muy favorable, desapareciendo los síntomas de insuficiencia respiratoria crónica y la necesidad de oxigeno adicional dentro de los primeros meses post alta. El requerimiento de oxigeno luego del alta es en general de 0,1 l/ min por cánula nasal durante 4,5 meses (+- 5 meses) (30). La mediana del tiempo de requerimiento de oxigeno luego de las 40 semanas es de 2,5 meses y el Percentil 75 de 8,5 meses (31). Las estrategias para dar de alta del 02 a lactantes con DBP son diferentes en distintos centros del mundo, utilizándose la saturometría continua en solo un $50 \%$ de los centros en un estudio en Estados Unidos (32). Los lactantes portadores de DBP moderada deben ser controlados mensualmente mientras tengan requerimiento de oxígeno, luego cada 2 meses hasta el año, semestralmente hasta los 2 años y anualmente hasta al menos los 7 años. En el caso de pacientes con DBP leve, sin requerimiento de oxígeno, deben ser controlados semestralmente hasta al menos los 7 años (14). Estas propuestas de control pueden cambiar según el compromiso clínico y de su función pulmonar. 
Los niños más graves, que requieren ventilación mecánica (VM) crónica, generalmente tienen daño neurológico por hemorragia endocraneana, necesitan gastrostomía, tienen mayor frecuencia de hipertensión pulmonar, y una mortalidad de hasta un 20\% (33). En un estudio de seguimiento de niños con DBP dependientes de VM, el 83\% pudieron salir de la VM, la mayoría antes de los 5 años, con una media de 2 años. El intervalo entre el destete de la VM y la decanulación fue de 11 meses (33).

La función pulmonar en niños con antecedente de DBP está alterada en mayor medida que en aquellos con antecedente de prematurez sin DBP. Frecuentemente se encuentra una reducción del Volumen espirado al primer segundo (VEF1) y reducción de la relación VEF1/CVF progresiva, mayor relación VR/CPT (Volumen residual/Capacidad pulmonar total), Io que implica atrapamiento aéreo, disminución de la difusión de monóxido de carbono (DLCO) e HRB (34)

Los niños con antecedente de nueva DBP pueden manifiestar un cuadro clínico semejante al asma e intolerancia al ejercicio. En estos pacientes se ha observado Oxido Nitrico exhalado disminuido, lo que implica ausencia de inflamación eosinofilica, menor respuesta a broncodilatadores y menor porcentaje de atopía, por lo que se postula que sería una entidad diferente al asma, propia de la $\operatorname{DBP}(9,35)$. Si bien el niño con antecedente de DBP va realizando una recuperación de su función pulmonar, algunos no lo logran por completo, lo que los predispone a un mayor y más rápido deterioro de la función pulmonar en caso de ser expuestos a noxas como el tabaco, aumentando la predisposición a desarrollar enfermedad pulmonar crónica del adulto (35).

Es muy importante realizar pruebas de función pulmonar en cuanto el niño tenga la capacidad de hacerlo (3 a 4 años, si no presenta compromiso neurológico), lo que va a permitir dirigir objetivamente si es de utilidad indicar corticoides inhalados y broncodilatadores, y establecer un pronóstico más adecuado a largo plazo.

\section{CONCLUSIONES}

La nueva DBP está dada por la supervivencia de prematuros en etapa canalicular del desarrollo pulmonar, con un freno en la alveolarización y angiogénesis y menor superficie de intercambio gaseoso. Se define como el requerimiento de oxigeno por 28 días, y la necesidad de oxigeno o ventilación mecánica definen el grado de severidad. Las únicas herramientas terapéuticas eficaces son la oxigenoterapia, la correcta nutrición y evitar sobreinfecciones. El compromiso de la función pulmonar es de por vida en la mayoría de los casos, por lo que se debe controlar a estos pequeños periódicamente, realizar función pulmonar en forma precoz y educar tempranamente para evitar el tabaquismo en adolescentes y adultos.

Los autores declaran no presentar conflicto de interés.

\section{REFERENCIAS}

1. Sánchez Luna M, Moreno Hernando J, Botet Mussons F, Fernández Lorenzo JR, Herranz Carrillo G, Rite Gracia S, Salguero García E, Echaniz Urcelay I. Displasia broncopulmonar: definiciones y clasificación. An Pediatr 2013; 79:262

2. Palominos MA, Morgues M, Martinez F. Management of infants with Chronic Lung Disease of prematurity in Chile. Early Hum Dev 200; 81: 151-4

3. Bancalari A. Actualización en Presentación y Patogénesis de la Displasia Broncopulmonar. Rev Chil Pediatr 2009; 80 : 213-224 4. Jobe AH. The new bronchopulmonary dysplasia. Curr Opin Pediatr 2011;23:167-72

5. Northway WH Jr, Rosan RC, Porter DY. Pulmonary disease following respiratory therapy of hyaline membrane disease: bronchopulmonary dysplasia. N Engl J Med 1967; 276: 357-68 6. Zeitlin P, Nogee L. Etiology of Bronchopulmonary Dysplasia: Before Birth. Pediatr Allergy Immunol Pulm 2011; 24: 21-25

7. Gien J, Kinsella J. Pathogenesis and treatment of bronchopulmonary dysplasia. Curr Opinion Pediatr 2011; 23:305-313

8. Mourani P, Abman S. Pulmonary vascular disease in bronchopulmonary dysplasia.: pulmonary hypertension and beyond. Curr Opin Pediatr 2013;25: 329-337

9. Baraldi E, Filippone M. Chronic Lung Disease after Premature Birth. N Engl J Med 2007; 357:1946-55

10. American Thoracic Society Documents Statement on the Care of the Child with Chronic Lung Disease of Infancy and Childhood. Am J Respir Crit Care Med; 2003: 356-396

11. Jobe A, Bancalari E. NICHD/NHLBI/ORD Workshop Summary. Bronchopulmonary Dysplasia. Am J Respir Care Med 2001; 163: 1723- 1729

12. Bancalari E, Claure N. Definitions and diagnostic criteria for bronchopulmonary dysplasia. Semin Perinatol, 2006, 30: 164170

13. Fernández C, Fajardo C, Favareto M, Hoyos A, Jijón-Letort F, Carrera M, Yllescas M, Romero M. Oxygen dependency as equivalent to bronchopulmonary dysplasia at different altitudes in newborns $\leq 1500 \mathrm{~g}$ at birth from the SIBEN network Members of Iberoamerican Society of Neonatology network. J Perinatol. $2014 ; 34: 538-42$

14. MINISTERIO DE SALUD. Guía Clínica Displasia Broncopulmonar del Prematuro. Santiago: MINSAL, 2009. web.minsal.cl/portal/ url/item/721fc45c974a9016e04001011f0113bf.pdf

15. Ola Didrik Saugstad, Dagfinn Aune. Optimal Oxygenation of Extremely Low Birth Weight Infants: A Meta-Analysis and Systematic Review of the Oxygen Saturation Target Studies. Neonatol 2014;105:55-63

16. Poets CF. When do infants need additional inspired oxygen? A review of the current literature. Pediatr Pulmonol. 1998 ;26:424-8

17. Kotecha S, Allen J. Oxygen therapy for infants with chronic lung disease. Arch Dis Child Fetal Neonatal 2002; 87:F11-4 18. Primhak RA. Discharge and aftercare in chronic lung disease of the newborn. Semin Neonatol 2003;8:117-26 
19. Rhein L, Simoneau T, Davis J, Correia C, Ferrari D, Monuteaux M, Gregory M. Reference values of nocturnal oxygenation for use in outpatient oxygen weaning protocols in premature infants. Pediatr Pulmonol 2012;47:453-9

20. Brockmann PE, Poets A, Urschitz MS, Sokollik C, Poets CF. Reference values for pulse oximetry recordings in healthy term neonates during their first 5 days of life. Arch Dis Child Fetal Neonatal 2011;96:F335-8

21. Mandy G, Malkar M, Welty S, Brown R, Shepherd E, Gardner W, Moise A, Gest A. Tracheostomy Placement in Infants With Bronchopulmonary Dysplasia: Safety and Outcomes Pediatr Pulmonol 2013;48:245-249

22. Darlow BA, Graham PJ. Vitamin A supplementation to prevent mortality and short and long-term morbidity in very low birthweight infants. Cochrane Database Syst Rev 2007 0ct 17;CD000501

23. Nair V, Loganathan P, Soraisham AS. Azithromycin and other macrolides for prevention of bronchopulmonary dysplasia: a systematic review and meta-analysis. Neonatol 2014;106:337-47 24. Grier D, Halliday L. Effects of Glucocorticoids on Fetal and Neonatal Lung Development Treatments. Respir Med 2004, 3: 295-306

25. American Academy of Pediatrics, Canadian Paediatric Society (fetus and newborn committee). Postnatal corticosteroids to treat or prevent chronic lung disease in preterm infants. Pediatr 2002; 109: $330-38$

26. Onland W, Offringa $M$, van Kaam $A$. Late ( $\geq 7$ days) inhalation corticosteroids to reduce bronchopulmonary dysplasia in preterm infants. Cochrane Database Syst Rev 2012 Apr 18;4:CD002311 27. Segar JL. Neonatal diuretic therapy: furosemide, thiazides, and spironolactone. Clin Perinatol. 2012; 39:209-20

28. Greenough A. Long term respiratory outcomes of very premature birth (<32 weeks). Semin Fetal Neonatal Med 2012;17:73-6

29. Updated Guidance for Palivizumab Prophylaxis Among Infants and Young Children at Increased Risk of Hospitalization for Respiratory Syncytial Virus Infection. Pediatr 2014, 134: 415-42 30. Hudak B, Allen M, Hudak M, Loughlin G. Home Oxygen Therapy for Chronic Lung Disease in Extremely Low-Birth-Weight Infants. Am J Dis Child 1989; 143: 357- 360

31. Hennessy E, Bracewell M, Wood N, Wolke D, Costeloe K, Gibson A, Marlow N for the EPICure Study Group. Respiratory health in pre-school and school age children following extremely preterm birth. Arch Dis Child 2008;93:1037-1043

32. Palm K, Simoneau T, Sawicki G, Rhein L. Assessment of Current Strategies for Weaning Premature Infants From Supplemental Oxygen in the Outpatient Setting. Adv Neonatal Care 2011; 11:349-356

33. Cristea A, Carroll A, Davis S, Swigonski N, Ackerman V. Outcomes of Children With Severe Bronchopulmonary Dysplasia Who Were Ventilator Dependent at Home. Pediatr 2013; 132: 727-734

34. Fawke J, Lum S, Kirkby J, Hennessy E, Marlow N, Rowell V, Thomas S, and J. Stocks J. Lung Function and Respiratory
Symptoms at 11 Years in Children Born Extremely Preterm The EPICure Study. Am J Respir Crit Care Med 2010;182: 237-245 35. Carraro S, Filippone M, Da Dalt L, Ferraro V, Maretti M, Bressan S, El Mazloum D, Baraldi E. Bronchopulmonary dysplasia: the earliest and perhaps the longest lasting obstructive lung disease in humans. Early Hum De 2013; 89 Suppl 3:S3-5 